\title{
Lexis
}

Journal in English Lexicology

Book reviews | 2021

\section{John HUMBLEY, La néologie terminologique}

Lambert-Lucas, 2018, 472 pages

\section{Frédérique Brisset}

\section{(2) OpenEdition}

\section{Journals}

\section{Electronic version}

URL: https://journals.openedition.org/lexis/6174

DOI: $10.4000 /$ lexis.6174

ISSN: 1951-6215

\section{Publisher}

Université Jean Moulin - Lyon 3

\section{Electronic reference}

Frédérique Brisset, "John Humbley, La néologie terminologique", Lexis [Online], Book reviews, Online since 13 December 2021, connection on 25 December 2021. URL: http://journals.openedition.org/ lexis/6174 ; DOI: https://doi.org/10.4000/lexis.6174

This text was automatically generated on 25 December 2021.

\section{(c) (i) (9)}

Lexis is licensed under a Creative Commons Attribution-NonCommercial-NoDerivatives 4.0 International License. 


\title{
John HUMBLEY, La néologie terminologique
}

Lambert-Lucas, 2018, 472 pages

\author{
Frédérique Brisset
}

\section{REFERENCES}

La néologie terminologique. Lambert-Lucas, coll. « La lexicothèque », Limoges, 2018. ISBN : 978-2-35935-226-9, Prix : $40 €, 472$ pages.

1 Avec ce volume conséquent préfacé par Rita Termmerman, John Humbley présente, en 12 chapitres et près de 500 pages, une somme encyclopédique de ses travaux, conduits sur plus de vingt-cinq années, présentée très justement par l'éditeur comme la «première synthèse historique, géographique et méthodologique, tant empirique que théorique, de la création terminologique " ( $4^{\mathrm{e}}$ de couverture). Professeur émérite à Sorbonne Paris Université, spécialiste de terminologie, néologie et traduction scientifique, il conjugue ici ces trois champs de recherche pour offrir, dans une première partie permettant fort à propos de contextualiser sa monographie, un vaste panorama historique et un état de la science sur la néologie terminologique, en France, territoire initial de prédilection de la spécialité, et au plan international (où elle apparaît en anglais sous la dénomination term formation) - une bibliographie de 45 pages témoigne d'ailleurs de la richesse de la littérature grise sur le sujet dans nombre de pays; dans un second temps, l'auteur propose une problématisation des principes de formation des néonymes à partir de l'exploitation de plusieurs vocabulaires de spécialité pour éclairer les grands mécanismes lexicogéniques. C'est l'occasion d'ailleurs pour lui de souligner les progrès méthodologiques imputables à l'émergence des techniques d'extraction de corpus automatisées durant les dernières décennies.

2 Toutes les langues sont en effet soumises à la dynamique néologique du fait de leurs « besoins de nomination " (p. 399) («A new word is a solution to a problem. It answers a need - intellectual, experiential», constate fort pragmatiquement Hitchings 
[2009:13]), même si le processus peut procéder d'une motivation délibérée ou inconsciente suivant les cas, comme l'ont souligné, entre autres auteurs, Sajous, Josselin-Leray \& Hathout [2018:18] dans le numéro de Lexis consacré à la néologie lexicale et sémantique en anglais. Toutes sont aussi en contact, induisant une néologie de traduction par les transferts (emprunts et calques lexicaux et/ou morphologiques, entre autres) abordés en fin d'ouvrage (chapitre 11) qui participent de cette dynamique de construction de nouveaux lexiques.

3 Mais chaque langue a aussi ses procédés lexicogéniques internes, que Humbley classe selon plusieurs modèles (chapitre 4). Le modèle incrémental postule que les néologismes sont élaborés à partir de termes existants (p.102) et englobe donc la composition (au sens large puisqu'elle comprend acronymie, siglaison ou mots-valises par exemple) illustrée au chapitre 5, et la dérivation. Le schéma discursif, discuté au chapitre 6, s'inspire, lui, de la linguistique cognitive, car il est conçu sur l'hypothèse que les termes résultent de «diverses formulations à visées explicatives, argumentatives» dont certaines aboutiront à un figement lexical pour finalement intégrer le vocabulaire spécialisé (p.161), et inclut métaphores grammaticales et variation discursive. Le dernier modèle (chapitre 7), baptisé métaphorique (au sens large, englobant et se combinant parfois avec la métonymie), est le plus étudié par les chercheurs, et Humbley souligne la complexité à le circonscrire dans la perspective néonymique. Il plaide donc l'ouverture d'esprit et prône un modèle mixte, basé sur l'approche de Becker (p. 119-122), seul apte à « intégrer les autres approches ».

4 Dans chacun de ces cadres conceptuels, Humbley décrit précisément les procédés de formation de nouvelles unités lexicales, détaille leur intérêt et leurs limites, sans jamais verser dans la prescription. Il offre une mise en perspective large et de nombreux exemples pertinents pour montrer comment le lexique rend compte de l'innovation (p. 30), mais la sert aussi, fidèle à sa conception de la terminologie comme l'« une des branches fondatrices de la linguistique appliquée » (p. 394). Il n'élude pas non plus les problèmes méthodologiques quant à la délimitation des procédés rencontrés, l'un des paradoxes soulignés tenant bien évidemment au fait que l'étude de la néologie est majoritairement rétrospective, son étude imposant le plus souvent un retour aux sources lexicales en diachronie, avant leur fixation lexicographique: "No semantic form is timeless. When using a word, we wake into resonance, as it were, its entire previous history", affirme d'ailleurs Steiner [1998:24]. Humbley défend ainsi une vision de la néologie comme "interface entre l'ancien et le nouveau " plutôt que comme rupture historique (p. 225), qu'il illustre avec l'exemple tout à fait passionnant de la lexicogenèse liée à l'émergence des technologies d'enregistrement et reproduction sonores au XIXe siècle (p. 228-236).

5 Ce riche volume, émaillé de 35 tableaux explicatifs et pourvu d'un fort utile index de 10 pages des nombreux auteurs cités, s'emploie ainsi à décrire et analyser les principes néonymiques principalement en langues anglaise et française, mais il fait aussi des incursions dans les langues orientales et africaines ou celles dites minorisées (l'exemple des langues celtiques est exploré p. 305-307); il s'attache à comprendre leurs rôles et leurs fonctions en langue générale, mais surtout en langues professionnelles, techniques, industrielles et scientifiques dans des domaines bien établis (administratif, juridique, pharmaceutique, chimique, médical, économique notamment), mais aussi en construction, tels le commerce électronique et la nouvelle économie abordés au chapitre 12), dans leur gestion individuelle et collective, mise en évidence par les 
politiques linguistiques et nomenclatures officielles en la matière via la normalisation, à laquelle est dévolu le chapitre 9. La néologie, si elle concerne tous les champs lexicaux, est en effet particulièrement active dans les technolectes, où elle interroge d'autant plus le lien entre «termes et savoir " sur lequel s'opposent les hypothèses déterministe et cognitiviste (p. 224). Cette caractéristique justifie ainsi la volonté de Humbley d'en étudier la genèse et la portée discursive plus précisément dans la sphère terminologique, lui permettant de mettre à profit cette double spécialisation de chercheur universitaire, avec rigueur et organisation, comme en atteste le choix de ponctuer son propos de nombreux titres et sous-titres pour guider son lectorat, et d'offrir à la fin de chaque chapitre une conclusion synthétique à la fois récapitulative et prospective.

6 Cette dense monographie vient combler une lacune éditoriale. En mêlant théorisation et recherche appliquée, elle rend compte de multiples approches sans en exclure aucune, en soulignant les avantages et inconvénients de chacune, dont la pertinence est liée tant à leur contexte d'application qu'à l'évolution des outils à la disposition de la recherche, mais relève aussi de visions diversifiées de ce qu'est la néologie terminologique. Abondamment documenté et convoquant de multiples références bibliographiques, ce sera un précieux outil pour les lexicologues, terminologues et historiens de la langue, pour comprendre et approfondir, comme l'écrivait Jean Tournier [1993:6], «les faits lexicaux sous leur aspect dynamique, créateur, "génétique" ", très certainement appelé à devenir un ouvrage de référence majeur en la matière, pour les chercheurs comme pour les professionnels de la normalisation terminologique et l'aménagement et la planification linguistiques.

\section{BIBLIOGRAPHY}

HITCHINGS Henry, 2009 [2008], The Secret Life of Words, How English Became English, London: John Murray.

SAjous Franck, JosSELIN-LeRAy Amélie \& Hathout Nabil, “The Complementarity of Crowdsourced Dictionaries and Professional Dictionaries viewed through the Filter of Neology", Lexis 12, 2018, https://doi.org/10.4000/lexis.2322

STEINER George, 1998 [1975], After Babel, Aspects of Language and Translation, Oxford: Oxford University Press.

TOURNIER Jean, 1993, Précis de lexicologie anglaise, Paris : Nathan.

\section{AUTHOR}

\section{FRÉDÉRIQUE BRISSET}

Frédérique Brisset, EA CECILLE, Université de Lille-SHS, France. 
Spécialiste de traduction et traductologie (anglais-français), Frédérique Brisset est docteure en études anglophones de l'Université Sorbonne Nouvelle et Maîtresse de Conférences honoraire à l'Université de Lille. Elle mène sa recherche en traduction audiovisuelle, traductologie, lexicologie contrastive, et stylistique. Elle a co-dirigé avec Lucile Bordet le numéro 17 de Lexis, « Humour, créativité et création lexicale »; ses derniers travaux portent sur l'usage des pérégrinismes comme inscription de la non-fiction dans la fiction (Palimpsestes 37, à paraître) et le doublage du lexique culinaire au cinéma (colloque Cecille, Lille, 2021). 